\title{
Desenvolvimento do Sistema de Desafios Abertos Inteligentes - IOCS: apresentação de um modelo inicial de Padrão Construtivista de Mediação ${ }^{\text {i }}$
}

\section{Development of the System of Intelligent Open Challenges - IOCS: presentation initial model of Standard Construtivista of Mediation}

\author{
Othon Bastos Filho \\ Programa de Pós Graduação em Informática na Educação - PGIE/UFRGS \\ Laboratório de Estudos em Linguagem, Interação e Cognição \\ othonb@hotmail.com \\ Paloma Dias Silveira \\ Faculdade de Educação - FACED/UFRGS \\ Laboratório de Estudos em Linguagem, Interação e Cognição \\ paloma.dias@gmail.com \\ Margarete Axt \\ Programa de Pós Graduaçao em Educação - PGEDU/UFRGS e Programa de Pós \\ Graduação em Informática na Educação - PGIE/UFRGS \\ Laboratório de Estudos em Linguagem, Interação e Cognição \\ maaxt2002@yahoo.com.br
}

\begin{abstract}
Resumo: O Padrão Construtivista de Mediação - PCM, apresentado neste artigo, é a formalização de um conjunto de categorias organizadoras de ações de agentes inteligentes, durante a interação com o Sistema Multiagentes Inteligent Open Challenges System- IOCS. Estas categorias são obtidas a partir do diálogo do Agente Estrategista (AE) com o Usuário (U) e têm como propósito tornar os agentes capazes de escolher a intervenção adequada para um possível desencadeamento de pertubações cognitivas por parte dos usuários. Desta forma, ao padronizar as ações dos agentes inteligentes, o PCM permitirá, também, a formalização do diálogo de agentes em construção no IOCS, como, em demais Sistemas Inteligentes.
\end{abstract}

Palavras-Chave: Método Clínico Piagetiano, Desafios Abertos, Sistemas Multiagentes e Agente Estrategista

Abstract: The Standard Construtivista of Mediation - PCM, presented in this article, is a set of categories organizers of action of intelligent agents, during the interaction with the System Multiagentes Inteligent Open Challenges System- IOCS. These categories are gotten from the dialogue of Agent Strategist (AE) with the User (U) and have as intention to become the agents capable to choose the intervention adjusted to cause cognitivas perturbations on the part of the users. In such a way, when standardizing the actions of the intelligent agents, the PCM will allow, also, the dialogue of agents in construction in the IOCS, as, in too much Intelligent Systems.

Word-Key: Clinical Method Piagetiano, Opened Challenges, Systems Multiagentes and Agent Strategist 


\section{Introdução}

O Padrão Construtivista de Mediação - PCM é a formalização de um conjunto de categorias organizadoras de ações de agentes inteligentes, durante a interação com o Sistema Multiagentes: Inteligent Open Challenges System - IOCS. Estas categorias são obtidas a partir do diálogo do Agente Estrategista (AE) com o Usuário (U) e têm como propósito tornar os agentes capazes de escolher a intervenção adequada para um possível desencadeamento de pertubações cognitivas por parte dos usuários. Desta forma, ao padronizar as ações dos agentes inteligentes, o PCM permitirá, também, a formalização do diálogo de agentes em construção no IOCS, como, em demais Sistemas Inteligentes (SILVEIRA, BASTOS FILHO e AXT, 2006a). ${ }^{\text {ii }}$

Ao propor um trabalho científico-pedagógico com a utilização de jogos, devemos considerar o diálogo como de fundamental importância para a criação de situações que instiguem o sujeito a pensar analiticamente sobre sua atividade, tomando consciência de suas ações. De acordo com Piaget (1978), esse processo de tomada de consciência consiste na passagem, por meio de regulações ativas, de esquemas de ação inconscientes para o nível da consciência, em diferentes graus, no sentido de uma (re) construção cognitiva, produzindo-se uma conceituação sobre a ação. Ocorre, nesse caso, também em diferentes graus, uma compreensão dos mecanismos internos envolvidos na ação, ou seja, o reconhecimento dos meios empregados para alcançar determinados objetivos, dirigindo-se da periferia para o C (centro do sujeito) e C' (centro do objeto), de forma correlativa.

Nos jogos de regras, a observação dos procedimentos adotados durante a interação possibilita a visualização, investigação e, posterior compreensão dos modos de estruturação do pensamento do sujeito (criança, jovem ou adulto); tornando, assim, possível a realização de situações problematizadoras, instigando a atividade cognitiva do sujeito. Nesse âmbito, pesquisadores como Macedo (2000) e Brenelli (1996) publicaram trabalhos em que afirmam a importância dos jogos para o desenvolvimento cognitivo, quando realizados em situações que permitem uma reflexão sobre o ato de jogar, a partir de uma metodologia de intervenções que desafiam e perturbam as estruturas cognitivas do jogador, estimulando-o a resolver situações-problema, por meio de mecanismos de regulação compensatória de caráter construtivo-inventivo.

$\mathrm{Na}$ interação com os jogos propostos no sistema IOCS, as situações desencadeadoras dos processos de tomadas de consciência dos usuários são fundamentadas numa metodologia de ações e intervenções. Cada ação do Usuário (U), como resposta ao (S), é vista como possibilidade de novas interações pelo (AE), de onde se parte para construir conhecimento baseado no pressuposto de que situações-deintervenção podem desencadear perturbações nas estruturas cognitivas, propiciando processos de reflexão, por meio das assimilações e acomodações, que caracterizam o funcionamento do sistema cognitivo, entendido como um ciclo aberto para novas organizações do conhecimento. Em contrapartida, o (AE) propõe novas intervenções, perguntas e problematizações, visando perturbar as estruturas cognitivas do (U), em constante adaptação, por meio da Tomada de Consciência de suas ações.

Porém, o diferencial deste (S) é que ele opera com agentes inteligentes, que têm como função realizar as intervenções, e simultaneamente, analisar as condutas cognitivas alfa $(\alpha)$, beta $(\beta)$ e gama $(\gamma)$ do $(U)$, traçando um mapa da evolução das tomadas de consciência, e adaptando-se a essa evolução. Neste sentido, cada intervenção do (AE) é analisada de acordo com a resposta do (U), formalizada no 
Padrão Construtivista de Mediação - PCM, que alimentará continuamente as Bases de Questões do (S), com o objetivo de realizar novas intervenções, perguntas e problematizações, a partir da observação constante da atividade do (U).

\section{Um modelo inicial de PCM}

Com o intuito de construir categorias organizadoras das ações dos agentes inteligentes, tornando-os capazes de escolher as intervenções adequadas para desencader pertubações cognitivas nos Usuários (U), foi construído o primeiro modelo do Padrão Construtivista de Mediação - PCM, cuja metodologia está calcada no Método Clínico Piagetiano, em sua variação como Método Clínico Crítico, criado e utilizado por Jean Piaget em suas pesquisas em Epistemologia Genética (BASTOS FILHO. O; AXT, M.; LABIDI, S.; COSTA, J. S.; SILVEIRA, P. D.; MACHADO, C. T., 2005) (SILVEIRA, P. D.; BASTOS FILHO, O. C.; AXT, M.; THOMAS, A. R. 2006c).

Piaget e Inhelder, na pesquisa sobre "A representação do espaço na criança" (1993), dedicaram um capítulo ao estudo da construção da noção de ordem linear e de ordem cíclica, concluindo que estas noções iniciam pela coordenação da própria atividade do sujeito. Posteriormente, nas pesquisas sobre a abstração da ordem das relações espaciais (1995), Piaget explica as diferenças entre a abstração empírica e reflexionante, na leitura da ordem, concluindo que esta leitura é um produto da abstração reflexionante, ou seja, da coordenação das ações. Os estudos supracitados nos revelam importantes características sobre a construção da noção de ordem e a abstração da ordem, tornando-os complementares no entendimento das relações espaciais, sendo estes utilizados para compreender e potencializar o desenvolvimento dessas relações pelo Usuário (U), na resolução das provas de concepção lógica utilizadas pelo IOCS, para a criação do primeiro modelo de PCM.

A metodologia do Método Clínico Piagetiano tem se mostrado adequada para investigar regularidades nas condutas dos sujeitos em interação com objetos. Estas regularidades representam maneiras de estruturação do pensamento e com base nelas é possível a elaboração de categorias organizadoras das ações dos agentes no Intelligent Open Challenge System - IOCS que, por meio do Agente Estrategista (AE), atribuem ao (S) a capacidade de realizar o mapeamento dos processos cognitivos do (U). Estes agentes, ao utilizar as ações do (U) na atividade proposta e informações prévias sobre os possíveis modos de organização desta atividade, definem as categorias específicas para cada $(\mathrm{U})$ construindo o PCM.

O PCM inclui todas as categorias e respectivas intervenções para um determinado jogo, situando-se na zona (ZAR) entre as ações do (U) e as ações do (S). Toda a ação do (U) gera uma categoria, e toda a categoria, dentro do PCM, corresponderá a um tipo específico de intervenção, a ser utilizada pelo (S), adequada ao estado cognitivo do (U) no momento de sua ação. As intervenções, por sua vez, também são elaboradas com base na Metodologia Clínica, a partir da análise das interações, durante a investigação clínica para construção do PCM do jogo (intervenções do experimentador, ações e respostas verbais do Usuário). Verificam-se então, quais intervenções foram mais eficientes no desencadeamento de processos de desequilíbrio e posterior tomada de consciência pelos (U). Após essa verificação, as intervenções são 
integradas ao (S) em formato de mensagens, que podem ser enviadas ao (U) por meio de uma caixa de texto ${ }^{2}$.

A partir da análise das filmagens ${ }^{3}$ realizadas na fase de criação do $(S)$ e da revisão teórica sobre os níveis de estruturação no desenvolvimento das noções espaciais relativas à ordem, bem como dos estágios de abstração reflexionante, na sua leitura, detectamos as sete estruturas de seqüências e a linguagem inicial das intervenções contidas nos perfis no $\mathrm{PCM}^{4}$.

\section{Desenvolvimento das Interfaces do PCM}

A primeira etapa para construção do Padrão Construtivista de Mediação PCM é a de desenvolvimento das interfaces do jogo. As interfaces servem de objeto de pesquisa para o Agente Estrategista (AE), ao serem interagidas pelos Usuários (U), e, concomitantemente, para o experimentador que utilizando a metodologia clínica, realiza intervenções na busca de compreender as hipóteses dos (U) e a organização de suas ações no jogo ${ }^{5}$ (SILVEIRA et al, 2006b).

Nesta pesquisa foi realizada uma adaptação da prova "A condição da leitura de séries aditivas complexas" (PIAGET, 1995), consistindo na apresentação de uma sequiência, ordenada segundo a lei AMAAMMAMAA..., e algumas variações das técnicas de investigação sobre a noção de ordem (PIAGET, 1993).

No Intelligent Open Challenge System - IOCS as interfaces (no total de seis) apresentarão a prova ao (U). Esta prova consiste na representação da ordem das seqüências, utilizando quatro elementos (círculos, segmentos, figuras geométricas e números) a serem aplicados de acordo com as fases observar, continuar e generalizar.

Na fase observar, o (U) observará a sequiência dada, com o objetivo de sua iniciação, nos processos de leitura da ordem. Com esta ação, o (U) deverá "observar" para "continuar" a sequiência.

$\mathrm{Na}$ fase continuar será enviada uma mensagem ao (U) para a continuação da seqüência, com o objetivo da experimentação de estratégias para a realização da tarefa. Com as ações de "continuar" as sequiências, o (U) poderá "generalizar" suas noções a respeito de suas ações, e o $(\mathrm{S})$ irá registrar as noções do $(\mathrm{U})$, a respeito da continuação da ordem, gerando, assim, um mapa de seus processos cognitivos ativados ${ }^{6}$.

$\mathrm{Na}$ fase generalizar serão apresentados elementos diversos, com o objetivo do entendimento pelo (U) da ordem apresentada nas sequências anteriores, podendo ser aplicada a outros conteúdos na criação de novos desafios, e o registro pelo $(\mathrm{S})$ de suas ações na leitura da ordem.

Depois de concluída a elaboração das interfaces, iniciam-se as filmagens com os (U) para a construção das categorias iniciais do PCM. 


\section{Categorias iniciais do PCM}

As estruturas de sequência elaboradas pelos Usuários (U), a partir das observações encontradas nas filmagens, representam as categorias iniciais do Padrão Construtivista de Mediação - PCM, de acordo com a tabela abaixo:

Tabela 1- Categorias iniciais do PCM. Fonte: SILVEIRA et al (2006c)

\begin{tabular}{|c|c|c|c|}
\hline Pesos & $\begin{array}{c}\text { Seqüência } \\
\text { proposta pelo } \\
\text { Sistema } \\
\end{array}$ & $\begin{array}{l}\text { Seqüência } \\
\text { prevista para o } \\
\text { Usuário } \\
\end{array}$ & Estruturas de Seqüências \\
\hline 0 & A A B A B B ... & A A B A B B ... & Seqüência Prevista \\
\hline 1 & А A B A B B ... & A A B B A A B B & $\begin{array}{l}\text { Seqüência desviada com alternâncias } \\
\text { duplas }\end{array}$ \\
\hline 2 & A A B A B B ... & A B A B A B ... & $\begin{array}{l}\text { Seqüência desviada com alternâncias } \\
\text { simples }\end{array}$ \\
\hline 3 & A A B A B B ... & A A A B B ... & $\begin{array}{l}\text { Seqüência desviada sem alternâncias } \\
\text { simples ou nem duplas }\end{array}$ \\
\hline 4 & A A B A B B ... & $\begin{array}{l}\mathrm{C} \mathrm{C} \mathrm{A} \mathrm{C} \mathrm{A} \mathrm{A} \ldots \\
\text { ou } \\
\mathrm{C} \mathrm{C} \mathrm{A} \mathrm{A} \mathrm{C} \mathrm{C} \ldots \\
\end{array}$ & $\begin{array}{l}\text { Seqüência com a utilização de dois } \\
\text { elementos não contidos na seqüência } \\
\text { original }\end{array}$ \\
\hline 5 & А A B A B B ... & B C D O D B C ... & $\begin{array}{l}\text { Seqüência com a utilização de mais de } \\
\text { dois elementos não contidos na } \\
\text { seqüência original }\end{array}$ \\
\hline 6 & A A B A B B & & Seqüência ausente \\
\hline
\end{tabular}

O Peso zero (0) é o mais próximo da estrutura de seqüência correta e o peso cinco (5) é o mais divergente da estrutura inicial de sequiência proposta pelo (S), no desafio.

De acordo com o funcionamento do PCM, para cada uma dessas categorias haverá tipos específicos de intervenção, selecionados de acordo com os processos cognitivos ativados pelo (U) durante o jogo.

Após a pesquisa inicial das categorias, parte-se para a análise da elaboração das intervenções. Essa é uma etapa exigente de novos testes, com o fim de estudar a linguagem mais adequada durante a interação (expressões, palavras, entre outras), para posteriormente organizá-las nas respectivas Bases de Intervenções ${ }^{7}$. Estas intervenções têm como objetivo desencadear perturbações cognitivas no Usuário (U), a fim de que ele coordene as suas ações visualizando a ordem; para este propósito, as intervenções não devem ser informadas ao (U), como "instrução", durante a interação.

O presente trabalho consiste na construção do IOCS, ressaltando a importância da linguagem para a intervenção do Sistema (S); porém, neste momento, não é foco deste estudo a análise da linguagem adequada na intervenção.

\section{Perfis no PCM}


Os perfis no Padrão Construtivista de Mediação - PCM são elaborados a partir das estruturas de seqüências obtidas pela ação do Usuário (U), juntamente com a linguagem inicial das intervenções propostas pelo Sistema (S), observadas nas filmagens, e adaptadas de acordo com o Método Clínico Piagetiano, em "A Representação do Mundo na Criança” (1926).

Na tabela 4 será realizada uma descrição dos perfis no PCM, analisando cada ação executada pelo (U), observada na filmagem, e a representação desta ação para o (S).

Tabela 2 - Descrição do Perfil no PCM. Fonte: SILVEIRA et al (2006c)

\begin{tabular}{|c|c|c|}
\hline $\begin{array}{c}\text { Ação do (U) observada na } \\
\text { filmagem }\end{array}$ & $\begin{array}{l}\text { Representação da ação do (U) para o } \\
\text { (S) }\end{array}$ & Perfis no PCM \\
\hline $\begin{array}{l}\text { Resposta imediata correta, } \\
\text { após a observação do desafio. }\end{array}$ & $\begin{array}{l}\text { Entendimento do desafio proposto. Não } \\
\text { há necessidade de intervenção pelo (S). }\end{array}$ & Espontânea \\
\hline $\begin{array}{lr}\text { Resposta desencadeada pela } \\
\text { intervenção } & \text { do } \\
\text { Experimentador. } & \end{array}$ & $\begin{array}{l}\text { Não há entendimento do desafio proposto. } \\
\text { Há a necessidade da primeira intervenção } \\
\text { pelo (AE) para propiciar a sistematização } \\
\text { do entendimento na direção da ordem. }\end{array}$ & Desencadeada \\
\hline $\begin{array}{llr}\text { Resposta desencadeada } & \text { por } \\
\text { várias intervenções } & \text { do } \\
\text { Experimentador. } & \end{array}$ & $\begin{array}{l}\text { Não há entendimento do desafio proposto } \\
\text { com a primeira intervenção do (AE). Há a } \\
\text { necessidade de nova intervenção do (AE), } \\
\text { sugestionando o entendimento da ordem. }\end{array}$ & Sugerida \\
\hline \begin{tabular}{llrr|} 
Resposta do (U) & não expressa \\
relação com & o & desafio \\
proposto. & & & \\
\end{tabular} & $\begin{array}{l}\text { Após várias intervenções pelo (AE), a } \\
\text { resposta do (U) discorda da ordem do } \\
\text { desafio. Há a necessidade de aplicação de } \\
\text { novo desafio para o (U). }\end{array}$ & Fabulada \\
\hline $\begin{array}{l}\text { Não há esforço de resposta } \\
\text { para o desafio proposto, } \\
\text { podendo o }(\mathrm{U}) \text { demonstrar-se } \\
\text { aborrecido. }\end{array}$ & $\begin{array}{l}\text { Não há interação com o (S), após um } \\
\text { determinado tempo. }\end{array}$ & Não-importista \\
\hline
\end{tabular}

\section{Sistematização das Ações e Intervenções no PCM}

De acordo com a revisão teórica, filmagens realizadas com grupos de alunos do Ensino Fundamental e análises de experimentadores ligados à pesquisa, foram sistematizadas as ações e intervenções do Intelligent Open Challenge System - IOCS ao Usuário (U) na tabela 5 (BASTOS FILHO et al, 2006a).

Tabela 3 - Sistematização das ações e intevenções ${ }^{8}$. Fonte: BASTOS FILHO et al (2006a) 


\begin{tabular}{|c|c|c|c|c|c|}
\hline \multicolumn{5}{|c|}{ SISTEMATZAÇÄO DAS AÇÖES E INTERVENÇÖES D O SISTEMA IOCS } & \\
\hline Perfil/Reaçäo & Tipo de s equência/Categorias & Peso & Açäo do sistema & Intervençäo (frases) & \\
\hline \multicolumn{5}{|c|}{${ }^{*}$ Essa tabela é elaborada com base na seguinte sequência: AAMAMM. } & \\
\hline \multirow{8}{*}{ ESPONTÂNEA } & sequência prevista & $0 \mathrm{~A}$ & Lançar próximo desafio & $\begin{array}{l}\text { Qual será o próximo desafio? Agora obs } \\
\text { uma nova sequência! }\end{array}$ & erve \\
\hline & $\begin{array}{c}\text { Sequência desviada, mas com os elementos } \\
\text { previstos: }\end{array}$ & & & & \\
\hline & a) alternâncias duplas: AA, MM, AA, MM.... & $1 \mathrm{~A}$ & Lançar $1^{\circ}$ interv. (Banco A) & $\begin{array}{c}\text { Nessas posiçöes as bolinhas continuan } \\
\text { sequência que você observou? }\end{array}$ & a \\
\hline & b) alternâncias simples: AM, AM, AM & $2 \mathrm{~A}$ & Lançar $1^{\circ}$ interv. (Banco A) & $\begin{array}{l}\text { Nessas posiçöes as bolinhas continuan } \\
\text { sequência que você observou? }\end{array}$ & a \\
\hline & $\begin{array}{c}\text { c) Nẫo apresenta alternâncias simples nem } \\
\text { duplas; Ex.; } A, A, A, B, B\end{array}$ & $3 \mathrm{~A}$ & Lançar $1^{\circ}$ interv. (Banco A) & $\begin{array}{c}\text { Nessas posiçöes as bolinhas continuan } \\
\text { sequência que você observou? }\end{array}$ & a \\
\hline & $\begin{array}{c}\text { sequência com elementos que não estavam } \\
\text { na sequência original: }\end{array}$ & & & & \\
\hline & $\begin{array}{c}\text { d) utilizando só dois elementos; Ex.: C CAC } \\
\text { AA... Ou C CAAC C ... }\end{array}$ & $4 \mathrm{~A}$ & Lançar $1^{\circ}$ interv. (Banco A) & $\begin{array}{l}\text { Porque você utilizou essas duas cores } \\
\text { bolinhas? Elas continuam a sequência }\end{array}$ & \\
\hline & $\begin{array}{l}\text { e) utilizando mais de dois elementos; Ex.: B } \\
\text { C D OD B C... }\end{array}$ & $5 \mathrm{~A}$ & Lançar $1^{\circ}$ interv. (Banco A) & $\begin{array}{l}\text { Quantos tipos de cores de bolinhas vo } \\
\text { utilizou? Elas continuam a sequência: }\end{array}$ & \\
\hline \multirow{8}{*}{$\begin{array}{l}\text { DE SEIICADEADA } \\
\text { (Banco de } \\
\text { intervençốes "A") }\end{array}$} & sequência prevista & $0 \mathrm{~A}$ & Lançar próximo desafio & $\begin{array}{l}\text { Qual será o próximo desafio? Agora obs } \\
\text { uma nova sequência! }\end{array}$ & serve \\
\hline & $\begin{array}{c}\text { sequência desviada, mas com os elementos } \\
\text { previstos: }\end{array}$ & & & & \\
\hline & a) alternâncias duplas: AA, MM, AA, MM .... & $1 \mathrm{~B}$ & Lançar $2^{\circ}$ interv. (Banco B) & $\begin{array}{l}\text { Observa as bolinhas! A azul tem sempre } \\
\text { vizinha azul e outra vizinha vermelha }\end{array}$ & uma \\
\hline & b) alternâncias simples AM, AM, AM & $2 \mathrm{~B}$ & Lançar $2^{\circ}$ interv. (Banळ B) & $\begin{array}{c}\text { Observe as bolinhas! A azul é vizinha son } \\
\text { chas vermelhas? }\end{array}$ & mente \\
\hline & $\begin{array}{l}\text { c) Nẫo apresenta alternâncias simples nem } \\
\text { duplas; Ex; } A, A, A, B, B\end{array}$ & $3 \mathrm{~B}$ & Lançar $2^{\circ}$ interv. (Bano B) & $\begin{array}{c}\text { Observa as bolinhas! As aztis såo vizinh } \\
\text { vermelhas? Quantas vezes? }\end{array}$ & is das \\
\hline & $\begin{array}{c}\text { sequência com exementos que não estavam } \\
\text { na sequência orginat. }\end{array}$ & & & & \\
\hline & $\begin{array}{c}\text { d) utilizando só dois elementos Ex.: C C AC } \\
\text { A A... Ou C C A AC C ... }\end{array}$ & 4日 & Lançar $2^{\circ}$ interv. (Banoo B) & $\begin{array}{c}\text { Exi stembolinhas rosas e amarelas ma sec| } \\
\text { que você observou? }\end{array}$ & ưência \\
\hline & $\begin{array}{l}\text { e) utilizando mais de dois elementos Ex: } \mathrm{B} \\
\qquad C D O D B C \ldots\end{array}$ & $5 \mathrm{~B}$ & Lançar $2^{\circ}$ interv. (Bano B) & $\begin{array}{l}\text { Você pode contimuar a secpuência utiliza } \\
\text { mais de duas cores de bolinhas? }\end{array}$ & ndo \\
\hline
\end{tabular}

Na tabela acima, o ítem "Ação do Sistema" denota a ação do Agente Estrategista (AE) na busca do desafio respectivo à Categoria em que se encontra o Usuário (U), baseado em seu perfil e peso, a fim de intervir adequadamente. $\mathrm{O}$ desafio a ser proposto pelo (AE) encontra-se armazenado no Banco de Desafios do (S), seguindo o critério de códigos descritos na tabela 5.

Tabela 4- Códigos do Banco de Desafios

\begin{tabular}{|c|l|}
\hline Código & \multicolumn{2}{|c|}{ Tipo do Desafio } \\
\hline A & Círculos simples continuar \\
\hline B & Segrnentos simples continuar \\
\hline C & Figuuras geométricas simples continua \\
\hline D & Números simples continuar \\
\hline E & Figuras simples criar \\
\hline F & \\
\hline G & \\
\hline H & \\
\hline
\end{tabular}


Assim, o código "A" corresponde ao desafio com círculos simples, o código "B" corresponde ao desafio com segmentos simples, e assim sucessivamente.

A partir da tabela "sistematização das ações e intevenções" são gerados os algorítmos do IOCS, conforme a seqüência abaixo:

01) Se (Perfil - Reação = Espontânea) Então Faça

02) $\mathrm{Se}(\mathrm{Se} 0=$ Certa $)$ Então Faça Peso = 0; Lançar Próximo Desafio

03) Senão

04) $\mathrm{Se}(\mathrm{Se} 0$ = Errada) Então Faça

05) Se “AE” Então Faça Peso = 1A; Lançar $1^{\circ}$ Intervenção (Banco A); Fim-

se

06) Se "B" Então Faça Peso = 2A; Lançar $1^{\mathrm{a}}$ Intervenção (Banco A); Fim-se

Novas análises oriundas da pesquisa levaram à necessidade de aperfeiçoamento contínuo do IOCS, objetivado com a construção do segundo protótipo do Sistema (S) na linguagem JAVA, trabalhando de forma mais robusta a comunicação entre os agentes inteligentes. Esse protótipo encontra-se em fase desenvolvimento, ficando como sugestão de trabalhos futuros o seu aperfeiçoamento, por meio de novas testagens, de forma a aprimorar as expressões utilizadas pelo experimentador virtual e criar novas categorias de ações do Usuário (U), aumentando, com isso, a sensibilidade de adaptação do (S), em relação ao (U).

\section{Referências}

AXT, M. Tecnologia para Educação: um texto em construção. In: Informática na educação: teoria \& prática. Porto Alegre, PGIE/UFRGS. (3), 1, p. 51-62, set. 2000.

BASTOS FILHO, O. C.; LABIDI, S.; AXT, M.; FONSECA, L.C.; NASCIMENTO, E. Increasing Cognitive Earmings using Intelligent Open Challenge Learning Objects. The International Association of Science and Technology for Development, USA. , 2004a.

BASTOS FILHO, O. C.; AXT, M.; LABIDI, S.; SILVEIRA, P. D.; COSTA, N. S.; FONSECA, L. C. Sistema de Desafios Abertos Inteligentes: um incremento computacional lúdico para se trabalhar o problema da aprendizagem. In: Revista Novas Tecnologias na Educação, ISSN 1679-1916, 2005a.

BASTOS FILHO, O. C.; AXT, M.; LABIDI, S.; COSTA, J. S.; SILVEIRA, P. D.; MACHADO, C. T. Sistemas Multiagentes Aplicado a Desafios Lógicos Abertos: Uma Possibilidade de Interação Através do Incremento Lúdico. In: Revista Novas Tecnologias na Educação, ISSN 1679-1916, 2005 b.

BASTOS FILHO, O. C.; AXT, M.; LABIDI, S.; MACHADO, C. T. Intelligent Open Challenges System: A possibility of adaptation of Piaget Clinical Method in Multi- 
agents Systems. In: IADIS Virtual Multi Conference on Computer Science and Information Systems. Lisboa, PT., 2006a.

BASTOS FILHO, O. C.; AXT, M.; LABIDI, S.; SILVEIRA, P. D.; THOMAS, A. R. SISTEMA INTELIGENTE DE DESAFIOS ABERTOS IOCS: uma proposta de adaptação dos padrões do Método Clínico Piagetiano em Plataforma Multiagentes. In: Revista Novas Tecnologias na Educação, ISSN 1679-1916, 2006b

BRENELLI, Rosely Palermo. O jogo como espaço para pensar: a construção de noções lógicas e aritméticas. Campinas: Papirus, 1996.

CARRAHER, T. N. O Método Clínico: usando os exames de Piaget. São Paulo: Cortez, 1989.

GIRAFFA, L. M. M.; VICCARI, R. M. Estratégias de ensino em Sistemas Tutores Inteligentes modelados através da tecnologia de agentes. Revista Brasileira de Informática na Educação. IE/SBC. (6), 2, 1999.

JAQUES, P. A. Agentes de Software na Monitoração da Colaboração em Ambientes Telemáticos de Ensino. Dissertação (Mestrado) - PPGCC/PUCRS. Porto Alegre, 1999.

MACEDO, Lino de. Aprender com jogos e situações-problema. Porto Alegre: ArtMed, 2000 .

OLIVEIRA, F. M. Critérios de equilibração para Sistemas Tutores Inteligentes. Tese (Doutorado) - UFRGS/CPGCC. Porto Alegre, 1994.

PIAGET, J. A representação do mundo na criança. Rio de Janeiro: Record, 1926.

PIAGET, J. A Equilibração das Estruturas Cognitivas. Problema central do desenvolvimento. Trad. Álvaro Cabral. Rio de Janeiro: Zahar, 1976a.

PIAGET, J.; et al. A Tomada da Consciência. Trad. Edson B. de Souza. São Paulo: Melhoramentos e EDUSP, 1977. 211p.

PIAGET, J. Fazer e Compreender. Trad. Cristina L. de P. Leite. São Paulo: Melhoramentos; EDUSP, 1978. 186p.

PIAGET, J. Abstração Reflexionante: Relações lógico-aritméticas e ordem das relações espaciais. Trad. Fernando Becker e Petronilha G. da Silva, Porto Alegre: Artes Médicas, 1995.

SILVEIRA, P. D. Desenvolvimento das Interfaces Gráficas do Sistema Inteligente IOCS: estudo das possibilidades de informatização de uma Prova Piagetiana. XVII SIC - Salão de Iniciação Científica da UFRGS. Porto Alegre, 2005

SILVEIRA, P. D.; THOMAS, A. R.; BASTOS FILHO, O. C.; AXT, M. Padrão Construtivista de Mediação: estudo do desenvolvimento de noções infralógicas espaciais topológicas durante a interação entre usuários e um sistema multiagente, com vistas à fundamentação de ações colaborativas entres eles. $13^{\mathrm{a}}$ Jornada Nacional de 
Iniciação Científica da 58 . Reunião Anual da SBPC. Universidade Federal de Santa Catarina - UFSC. Florianópolis, SC, 2006b.

${ }^{\text {i }}$ Apoio da Fundação de Apoio e Âmparo à Pesquisa do Estado do Maranhão - FAPEMA

${ }^{2}$ A interface de administração do Sistema que permitirá a inclusão e configuração das mensagens, foi desenvolvida pelo bolsista Andrei R. Thomaz. No estágio atual, as mensagens são em formato de texto escrito; espera-se que posteriormente estas intervenções possam ser transmitidas por um mecanismo de viva voz.

${ }^{3}$ Realizadas com alunos cursantes da primeira série do ensino fundamental na Escola Municipal de Educação Básica Santa Rita de Cássia, Gravataí - RS. Ver ANEXOS A, B e C.

${ }^{4}$ As estruturas de seqüências e a linguagem inicial foram obtidas a partir da explanação oral dos alunos sobre as suas ações realizadas durante a intervenção do experimentador, registradas nas filmagens citadas anteriormente.

${ }^{5}$ É importante a filmagem desta atividade, a fim de que o experimentador obtenha um material com todas as informações possíveis de serem analisadas.

${ }^{6}$ Foram disponibilizadas variedades de elementos para que o usuário tenha opção de escolha no momento de continuar a seqüência. Esta é uma variação da prova, visando diminuir a possibilidade do usuário continuar corretamente ou construir alternâncias simples e/ou duplas, sem que tenha realmente conseguido realizar a leitura da ordem representada, simplesmente pela falta de opções na escolha de elementos.

${ }^{7}$ As Bases de Intervenção formam os perfís do IOCS.

${ }^{8}$ A tabela descrita continua com os perfís de Crença Sugerida, Fabulação e Não-Importismo. 